\title{
Electric field induced surface-enhanced Raman spectroscopy for multianalyte detection
}

Walia, Sumeet; Shah, Aditya; Stoddart, Paul; Bhaskaran, Madhu; Sriram, Sharath

https://researchrepository.rmit.edu.au/esploro/outputs/9921862150601341/filesAndLinks?institution=61RMIT_INST\&index=null

Walia, S., Shah, A., Stoddart, P., Bhaskaran, M., \& Sriram, S. (2015). Electric field induced surface-enhanced Raman spectroscopy for multianalyte detection. Physical Chemistry Chemical Physics, 17(11), 7095-7099. https://doi.org/10.1039/c4cp04912h

Document Version: Accepted Manuscript

Published Version: https://doi.org/10.1039/c4cp04912h 
Thank you for downloading this document from the RMIT Research Repository.

The RMIT Research Repository is an open access database showcasing the research outputs of RMIT University researchers.

RMIT Research Repository: http://researchbank.rmit.edu.au/

\section{Citation:}

Walia, S, Shah, A, Stoddart, P, Bhaskaran, M and Sriram, S 2015, 'Electric field induced surface-enhanced Raman spectroscopy for multianalyte detection', Physical Chemistry Chemical Physics, vol. 17, no. 11, pp. 7095-7099.

See this record in the RMIT Research Repository at:

https://researchbank.rmit.edu.au/view/rmit:30063

Version: Accepted Manuscript

Copyright Statement:

(C) the Owner Societies 2015

\section{Link to Published Version:}

http://dx.doi.org/10.1039/c4cp04912h 


\title{
COMMUNICATION
}

\section{Electric field induced surface-enhanced Raman spectroscopy for multianalyte detection}

Cite this: DOI: $10.1039 / \times 0 \times x 00000 x$

\author{
Sumeet Walia, ${ }^{a b \S}$ Aditya K. Shah, ${ }^{a b \S}$ Paul R. Stoddart, ${ }^{c}$ Madhu Bhaskaran ${ }^{a b}$ \\ and Sharath Sriram*ab
}

Received 00th January 2014,

Accepted 00th January 2014

DOI: $10.1039 / \times 0 \times x 00000 x$

www.rsc.org/

\begin{abstract}
This work demonstrates the ability to identify thiophenol from a mixture with structurally similar benzyl mercaptan. We exploit the unique alterations that occur in the surface-enhanced Raman spectroscopy (SERS) characteristics in the presence of an oscillating electric field applied using SERS-active microfabricated electrode pairs.
\end{abstract}

Highly sensitive and molecule-specific detection within a chemical mixture is of great interest, especially for applications in chemistry, biology, environmental science, and forensics. Raman spectroscopy relies on the examination of molecular vibrations and offers high selectivity due to its usually narrow, well-resolved vibrational bands that yield structural information of the analyte molecules. ${ }^{1,2}$ The field of surface-enhanced Raman spectroscopy (SERS), where a million-fold up to $10^{16}$ times enhancement in sensitivity is possible, was discovered nearly 30 years ago when an amplified spectrum of pyridine was detected on roughened silver. ${ }^{3,4}$ Since then, SERS has seen rapid developments to emerge as an attractive analytical tool for the detection of molecules that are attached to nanometresized metallic structures. ${ }^{5-8}$

SERS involves illumination of the analysis surface with focussed laser radiation that results in localized surface plasmon modes in metallic nanostructures. The nanostructure morphology creates electromagnetic field concentration or localisation to create "hot spots". ${ }^{9,10,11}$ Structures made of gold, silver, and copper are most typically used for SERS since they sustain plasmon resonances in the visible range. This technique has been widely employed to identify chemical species and obtain structural information in a wide range of fields like biochemistry, chemical manufacturing, and forensics. ${ }^{12,13}$
However, researchers are yet to achieve any major breakthrough in the field of SERS (or Raman spectroscopy for that matter) to discretely detect target molecules in a mixture with multiple analytes. ${ }^{14}$ The ability to detect and isolate multiple analytes will widen the range of applications of SERS, and potentially render it as a practical and widely adopted technique.

We have previously used the combination of microfabricated electrode pairs and silver nanotextures to investigate the influence of an external alternating current (AC) electric field on the SERS spectra of thiophenol molecules. The electric field was shown to selectively influence specific vibrational modes of the molecule, with a dependence on frequency and strength of the applied electric field. ${ }^{15}$

Here, we report the capability to identify thiophenol in a mixture of two analytes (thiophenol and benzyl mercaptan) using electrode pair integrated SERS microdevices. Thiophenol and benzyl mercaptan are highly popular materials in biological applications that include surface coatings, chemical sensors and medical devices. ${ }^{16}$ They are also the most common thiol catalysts. The molecular structures of the thiophenol and benzyl mercaptan are shown in Figs. $1 \mathrm{a}$ and $1 \mathrm{~b}$ respectively. Structurally, they are the simplest thiols only differentiated by an additional $\mathrm{C}-\mathrm{C}$ bond. Hence, we choose these two thiols as representative analytes for our work, with the aim of identifying and resolving such reasonably similar molecules.

An oscillating electric field in combination with SERS is used, which provides a multivariate external control capability. This allows the excitation of signature vibrational modes of the molecules which can be used for identifying the target analytes (thiophenol in this case) in a mixture. A comprehensive 
analysis of the frequency-dependent analyte vibration modes in the presence of an electric field is performed for the individual
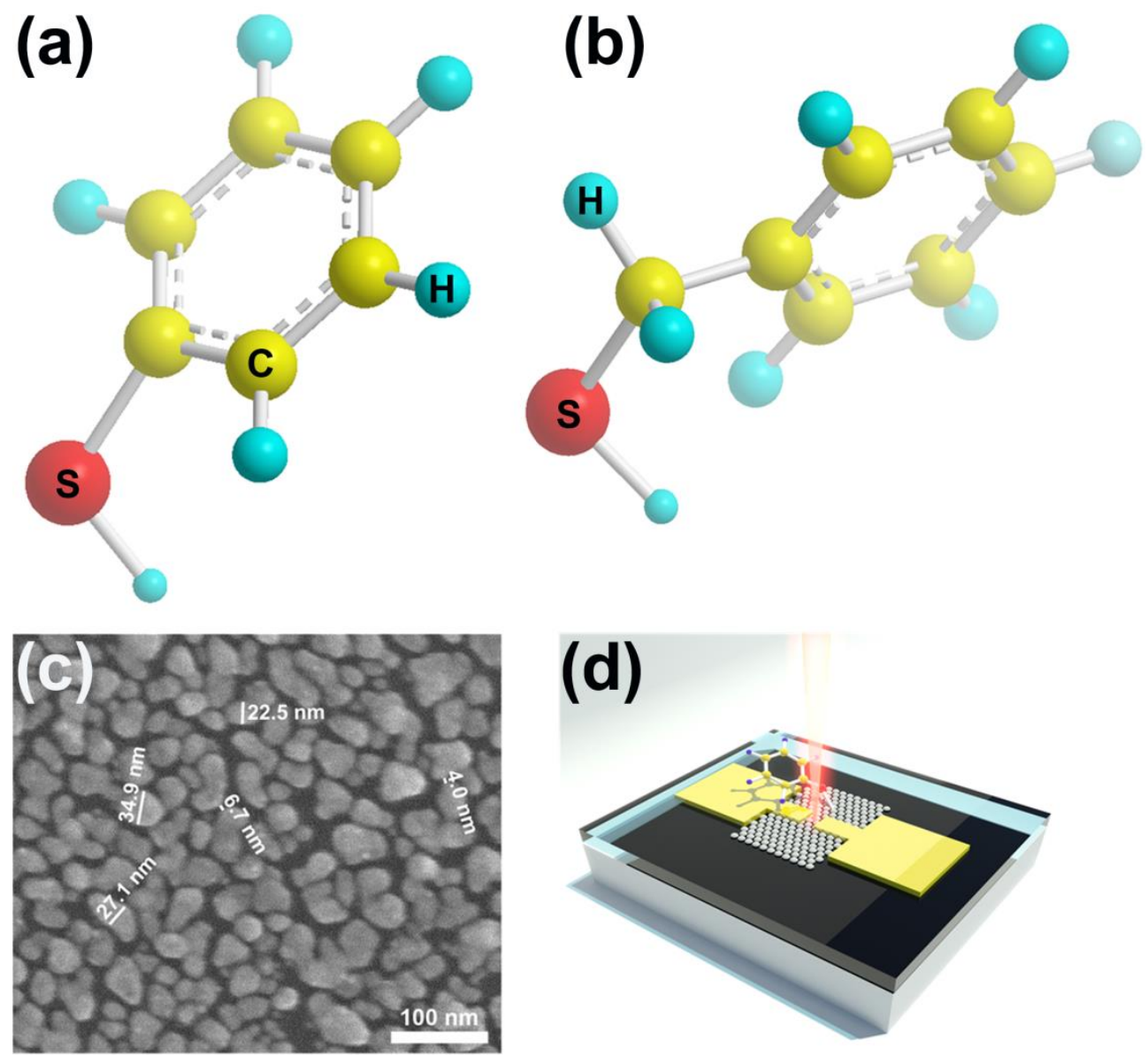

(d)

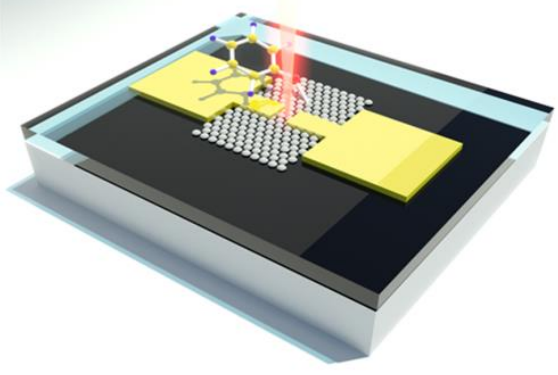

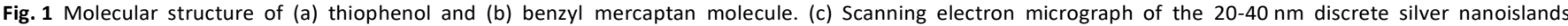
deposited by oblique angle deposition and (d) Artist's impression of a typical microelectrode pair device used in this study.

analytes. Subsequently, the distinct vibration mode behaviours are identified and used to decipher the target analyte from within a two-analyte 1:1 molar ratio mixture.

SERS-compatible silicon wafers were prepared by using photolithography and subsequently coated with silver using the oblique angle deposition (OAD) technique which resulted in discrete silver nanoislands about $20-40 \mathrm{~nm}$ in diameter. ${ }^{17}$ Figure 1c shows a scanning electron micrograph of the silver nanoislands and Fig. 1d depicts an artist's impression of the asfabricated microelectrode devices. The discrete nature of the silver nanoislands ensures that there is no current flow during the application of an external electric field. SERS measurements were performed using a Renishaw Raman spectrometer using a normally polarized $785 \mathrm{~nm}$ laser incident perpendicularly onto the surface with a $1.1 \mathrm{~mW}$ incident power, $10 \mathrm{~s}$ exposure, and a spot size of $\sim 1 \mu \mathrm{m}$. The excitation laser is positioned so that it is incident directly on the microelectrode gap by using the microscope and controllable stage associated with the Raman system. A microscope with a 50× objective was used.

Sinusoid electric fields oscillating between $\pm 20 \mathrm{~V}$ were applied using an waveform generator (Agilent Technologies,
USA) across the $2 \mu \mathrm{m}$ gap electrode pair devices that comprised of a $100 \mathrm{~nm}$ thick gold layer deposited on to $10 \mathrm{~nm}$ chromium which was used as the adhesion layer. This resulted in a peak electric field strength of $\pm 10 \mathrm{kV} / \mathrm{mm}$. Reference SERS spectra were collected with no external electric field which served as the control experiments. This data was used for normalizing the results acquired in the presence of an externally applied oscillating electric field. The resulting normalized intensities of peaks of interest were determined.

To start with, the SERS spectrum of individual thiophenol and benzyl mercaptan were obtained to ascertain the vibrational modes that are excited at this wavelength. This was carried out by dipping two separate microelectrode devices in a $10 \mathrm{mM}$ ethanolic solution of thiophenol and benzyl mercaptan, respectively, for $10 \mathrm{~min}$ and subsequently acquiring their Raman spectra. Thiophenol is a polar molecule that consists of a benzene ring with a sulphur tether (Fig. 1a) and is commonly used as a test analyte as sulphur usually forms a covalent bond with gold and silver, thereby providing a high degree of Raman scattering. Previous studies have shown that thiophenol stabilizes in an erect configuration that depends on the surface 
orientation and location of the molecule on the metal surface. ${ }^{18,19}$

Under the influence of an oscillating electric field, the thiophenol molecules realign with respect to the direction of the electric field. Figure 2 a shows the normalised Raman spectra obtained at different frequencies in the presence of a $\pm 10 \mathrm{kV} / \mathrm{mm}$ electric field. The reference spectrum (obtained in the absence of the electric field) is also shown.

Table 1: Assignment of vibrational modes for thiophenol Raman peaks. ${ }^{16}$ Only the peaks of interest are listed.

\begin{tabular}{|c|c|c|}
\hline \multicolumn{2}{|c|}{ Raman Shifts $\left(\mathrm{cm}^{-1}\right)$} & \multirow{2}{*}{$\begin{array}{l}\text { Vibration Mode } \\
\text { Assignments }\end{array}$} \\
\hline Theoretical & Experimental & \\
\hline 1018 & 1020 & $\begin{array}{l}\text { Ring in-plane deformation } \\
\text { and } \mathrm{C}-\mathrm{C} \text { symmetric } \\
\text { stretching }\end{array}$ \\
\hline 990 & 1000 & $\begin{array}{l}\text { Ring out-of-plane } \\
\text { deformation and C-H out-of- } \\
\text { plane bending }\end{array}$ \\
\hline 471 & 469 & C-S out-of-plane bending \\
\hline 404 & 417 & $\begin{array}{l}\mathrm{C}-\mathrm{S} \text { stretching and ring in- } \\
\text { plane deformation }\end{array}$ \\
\hline
\end{tabular}

The wavenumber and intensity of four representative peaks with different bond structure assignment were selected for analysis. ${ }^{16}$ These four peaks of thiophenol and their associated vibrational modes are presented in Table 1 . The trends of the relative peak intensities of the representative peaks at different frequencies normalised to the case of no electric field acquired at $785 \mathrm{~nm}$ excitation wavelength are shown in Fig. 2b. The magnitude of error in the acquired data is approximately of the order of $5 \%$. However, an accurate evaluation of error in every data point is complex. Nevertheless, the arguments made in the study are based on the frequency dependent trends in intensity ratios. It can be seen that the $469 \mathrm{~cm}^{-1}$ peak intensity sharply increases at $100 \mathrm{mHz}$ before decreasing at $1 \mathrm{~Hz}$. It follows a similar pattern throughout the acquired range. The $417 \mathrm{~cm}^{-1}$ peak too exhibits a similar trend but with a weaker intensity and becomes stable at frequencies over $1 \mathrm{kHz}$. Although the $417 \mathrm{~cm}^{-1}$ peak does not show very apparent changes under the $514 \mathrm{~nm}$ Raman laser wavelength reported previously, ${ }^{15}$ it starts showing atypical behaviour at a higher excitation wavelengths (Fig. 2b). The 417 and $469 \mathrm{~cm}^{-1}$ peak behaviours could be explained by the bond structure and symmetry causing the SERS effect. The $469 \mathrm{~cm}^{-1}$ peak represents the bending modes of the $\mathrm{C}-\mathrm{S}$ bond in thiophenol, while the $417 \mathrm{~cm}^{-1}$ peak relates to the bending and stretching modes of the $\mathrm{C}-\mathrm{S}$ bond in thiophenol (see Table 1). Additionally, the $1000 \mathrm{~cm}^{-1}$ (that represents $\mathrm{C}-\mathrm{H}$ bending) and the $1020 \mathrm{~cm}^{-1}$ (that represents $\mathrm{C}-$ C stretching) peaks show a gradually decreasing intensity with frequency and small oscillations starting to occur at frequencies larger than $100 \mathrm{~Hz}$. The complex response of the 417 and $469 \mathrm{~cm}^{-1}$ peaks can be ascribed to the continuous realigning of the net polarization of the thiophenol molecules with respect to the polarity of the externally applied oscillating electric field. The realignment of the benzene ring governs the molecular bonds that align with the polarization of the surface plasmon (a)

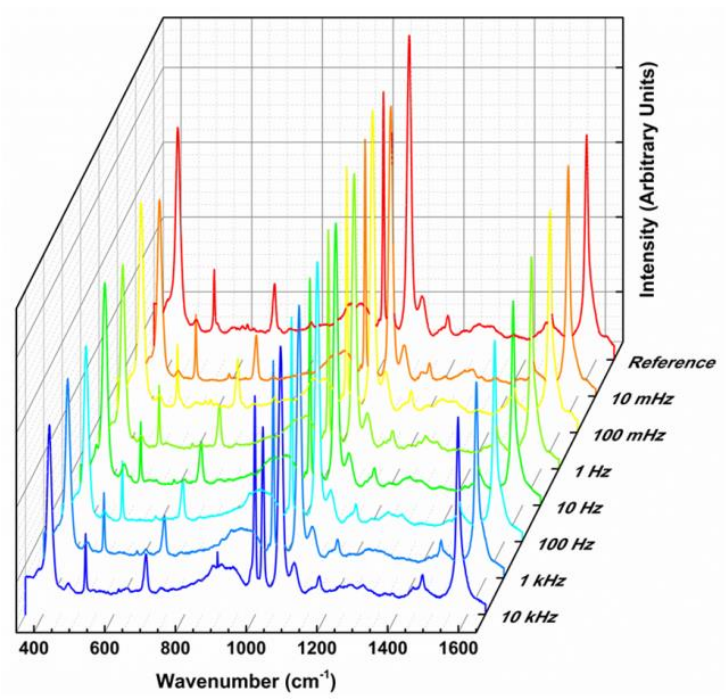

(b)

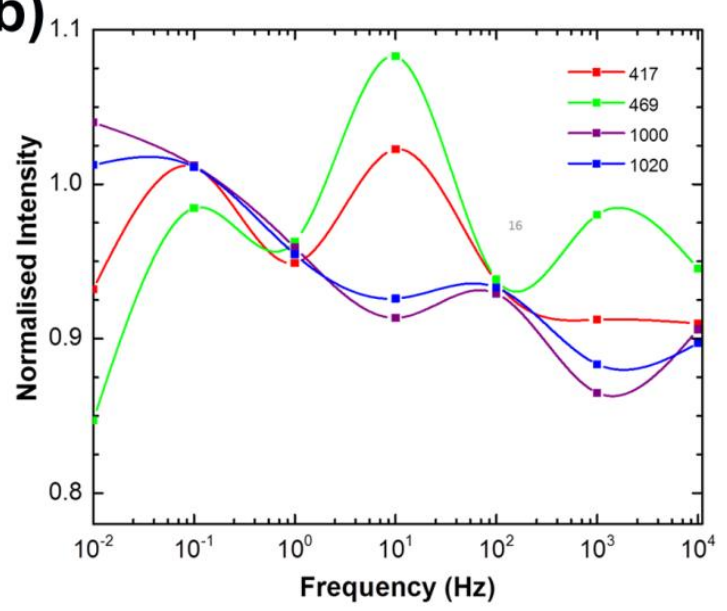

Fig. 2 (a) Raman spectra of thiophenol measured at different frequencies under a $\pm 10 \mathrm{kV} / \mathrm{mm}$ electric field (reference spectra under no electric field also shown). (b) The trends in peak intensities as a function of applied field frequency for four significant peaks normalised to the spectrum under no electric field. Trendlines are included as a visual guide.

excited by the incident laser. The maximum spectral response is for the bonds that align normal to the substrate surface based on the rules proposed by Suh et al. ${ }^{20}$ It should be noted that the band frequencies observed in these results can be slightly shifted from neat thiophenol due to the molecule binding to the metal and preferential alignment on the non-planar metallic nanostructure. ${ }^{21}$

Subsequent to establishing the signature peaks of thiophenol, the spectra for benzyl mercaptan were obtained under identical conditions and acquisition parameters (Fig. 3a). The corresponding normalised intensity ratios of the selected peaks of interest are shown in Fig. 3b (all peaks not shown for brevity). These peaks of benzyl mercaptan and their associated vibrational modes are presented in Table 2 . It can be seen that the $648 \mathrm{~cm}^{-1}$ peak exhibits a relatively low intensity compared to the other peaks. Similar to thiophenol, it was found that the asymmetric $\mathrm{C}-\mathrm{S}$ bond, which fixes thiophenol to a substrate, is most susceptible in benzyl mercaptan as well. Two peaks at 


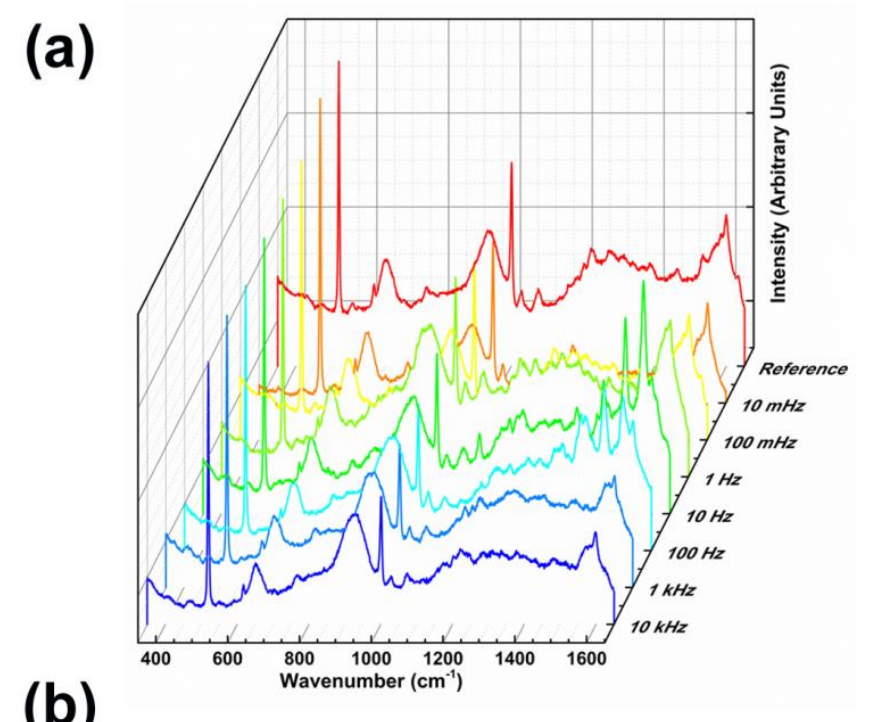

(b)

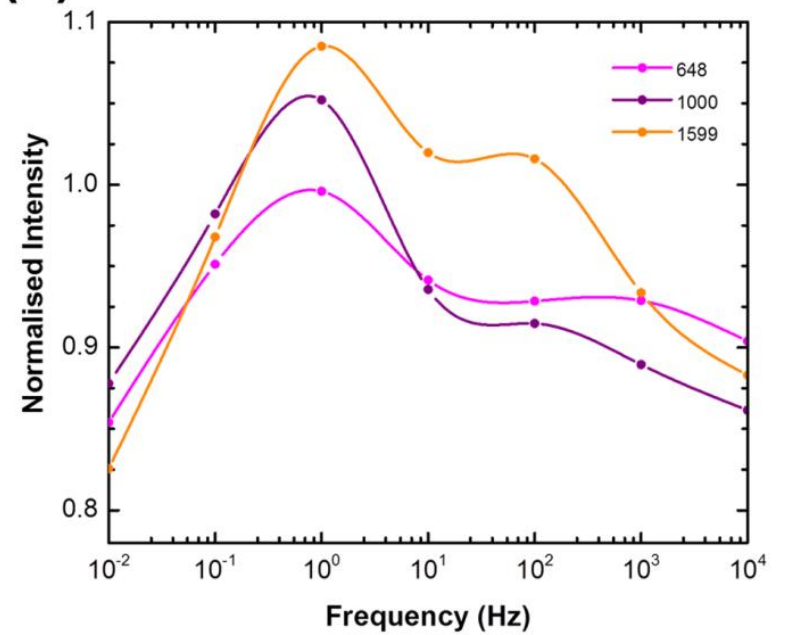

Fig. 3 (a) Raman spectra of benzyl mercaptan measured at different frequencies under a $\pm 10 \mathrm{kV} / \mathrm{mm}$ electric field (reference spectra under no electric field also shown). (b) The trends in relative peak intensity ratios of three significant peaks at different frequencies normalised to the case of no electric field. Trendlines are included as a visual guide.

648 and $1000 \mathrm{~cm}^{-1}$ represent $\mathrm{C}-\mathrm{S}$ stretching and $\mathrm{C}-\mathrm{H}$ wagging, respectively. The 648 peak behaviour can be attributed to the SERS effect due to the bond structure and symmetry. Excitation at $785 \mathrm{~nm}$ does not reveal the $1217 \mathrm{~cm}^{-1}$ peak that is typically seen at lower excitations and relates to the bending and twisting modes of the $-\mathrm{CH}_{2}$ bond of benzyl mercaptan. ${ }^{22}$

After ascertaining the individual characteristics of the two target molecules used in this study, the electrode pair sample was immersed in an equivalent molar mixture of thiophenol and benzyl mercaptan for $10 \mathrm{~min}$ in order to test the capabilities of our approach to identify the presence of an analyte in a mixture. The SERS spectra of the mixture normalised to the case of no electric field is shown in see Fig. 4a. The corresponding intensity ratios of selected Raman peaks are shown in Fig. 4b. It can be seen that the 417 and $469 \mathrm{~cm}^{-1}$ peaks (which are typical of thiophenol) follow a distinct trend compared to all the other peaks depicted in the plot.
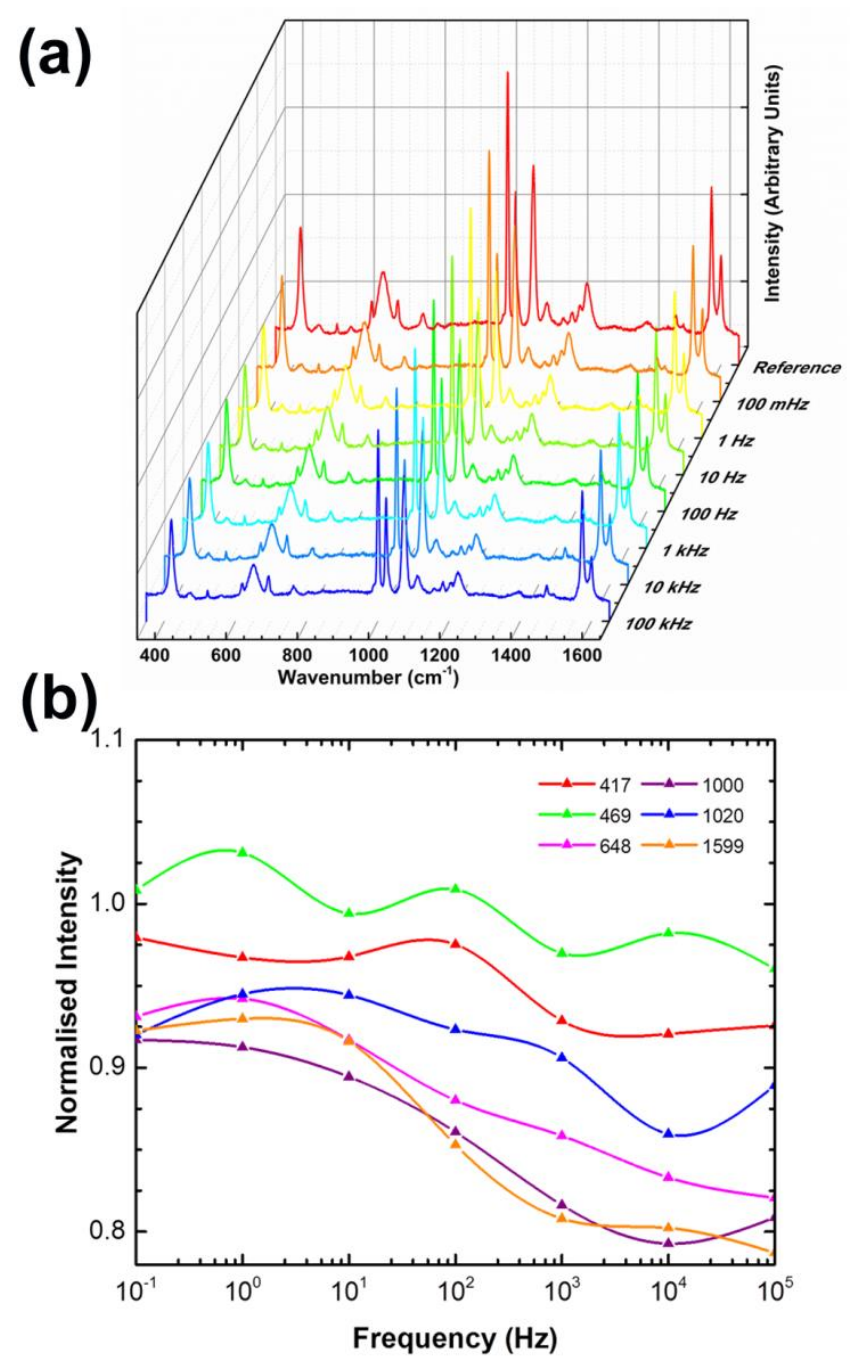

Fig. 4 (a) Raman spectra of a sample dipped in a 1:1 molar mixture of thiophenol and benzyl mercaptan at different frequencies under a $\pm 10 \mathrm{kV} / \mathrm{mm}$ electric field (reference spectra under no electric field also shown). (b) The trends in relative peak intensity ratios of the significant peaks at different frequencies normalised to the case of no electric field. Trendlines are included as a visual guide.

It has been shown in a previous study that specific bonds of thiophenol can be distinguished not only by the Raman shift but also from the relationship between the SERS field intensity and the externally applied electric field. ${ }^{15}$ The deformation of the $\mathrm{C}-\mathrm{S}$ bond and the consequent alignment alterations of the benzene ring directly affect the $417 \mathrm{~cm}^{-1}$ peak. As such, the bending of the $\mathrm{C}-\mathrm{S}$ bond and the subsequent deformations of the bound benzene ring under a strong applied field are interrelated. The distinctive behaviour of the fingerprint thiophenol peaks (417 and $469 \mathrm{~cm}^{-1}$ ) can be easily used to pinpoint the presence of this molecule in the mix--ture. This characteristic feature can be explained by considering the specific bond symmetry that causes SERS. As shown in Table 1, the $469 \mathrm{~cm}^{-1}$ peak represents the bending modes of the $\mathrm{C}-\mathrm{S}(-\mathrm{Ag})$ bond which tethers the thiophenol to the surface of the Ag nanostructures, while the $417 \mathrm{~cm}^{-1}$ peak represents the stretching of the $\mathrm{C}-\mathrm{S}$ bond as well as the benzene ring deformations. 
Table 2: Assignment of vibrational modes for benzyl mercaptan Raman peaks. ${ }^{23}$ Only the peaks of interest are listed.

\begin{tabular}{|c|c|c|}
\hline \multicolumn{2}{|c|}{ Raman Shift $\left(\mathrm{cm}^{-1}\right)$} & \multirow{2}{*}{$\begin{array}{l}\text { Vibration Mode } \\
\text { Assignments }\end{array}$} \\
\hline Theoretical & Experimental & \\
\hline 1597 & 1599 & $\begin{array}{l}\text { Aromatic } \mathrm{C}-\mathrm{H} \text { in-plane } \\
\text { bending and } \mathrm{C}-\mathrm{C} \text { ring } \\
\text { stretching }\end{array}$ \\
\hline 644 & 648 & $\begin{array}{l}\mathrm{C}-\mathrm{S} \text { stretching and ring in- } \\
\text { plane deformation }\end{array}$ \\
\hline 997 & 1000 & Out-of-plane $\mathrm{C}-\mathrm{H}$ wagging \\
\hline
\end{tabular}

Such bond bending or ring deformation dependent changes have been previously observed in bending kinetics induced plasmon resonances in DNA. ${ }^{24,25}$ These results also highlight a strong correlation of the spectral alterations to the electric fieldinduced molecular realignments. Density functional theory (DFT) studies conducted on isolated thiophenolates adsorbed on to low-index, defect-free Ag (111) surface have also shown that the $\mathrm{S}-\mathrm{Ag}$ bond length reduces when an electric field is applied, while the adsorption energy also increases. ${ }^{15} \mathrm{~A}$ combination of the aforementioned factors can explain the typically distinctive behaviour of the 417 and $469 \mathrm{~cm}^{-1}$ peaks observed for a large applied field $( \pm 10 \mathrm{kV} / \mathrm{mm}$ in this case $)$ in Fig. 4b.

In summary, we have demonstrated the ability to detect the presence of a thiophenol molecule in a 1:1 molar mixture with benzyl mercaptan using a SERS-active novel microelectrode pair device. The behaviour of selected peaks at different frequencies under an applied electric field is used to distinguish the target molecule. It is shown that the specific bonds can be distinguished not only by the Raman shifts but also their dependence on frequency under the influence of an oscillating electric field. Although the effectiveness of this method has to be tested for different target molecules, it is clear that in the presence of an oscillating electric field, some peaks (depending on the bond alterations) exhibit distinct behaviours that are unique to that particular molecule. These distinctly identifiable characteristics can be used to distinguish the presence of the target molecule. As such, the ability to manipulate the Raman spectra dynamically can potentially enable highly selective analysis of complex mixtures that contain multiple analytes to decipher target molecules. Such a capability can have far reaching consequences in biochemistry, medicinal, and forensic applications.

\section{Acknowledgements}

The authors acknowledge financial support from the Australian Research Council via fellowship (DP1092717 and DP110100262), project (DP110100262 and DP130100062), and infrastructure (LE100100215) funding. The authors also acknowledge Jennifer Hartley and Naveed Ahmed for technical assistance.

\section{Notes and references}

a Functional Materials and Microsystems Research Group, RMIT University, Melbourne, Australia.

${ }^{b}$ MicroNano Research Facility, RMIT University, Melbourne, Australia.

${ }^{c}$ Faculty of Engineering and Industrial Science, Swinburne University of Technology, Hawthorn, Australia.

$\S$ These authors contributed equally to this work.

1. W. E. Doering, M. E. Piotti, M. J. Natan and R. G. Freeman, $A d v$. Mater., 2007, 19, 3100-3108.

2. X. M. Qian and S. M. Nie, Chem. Soc. Rev., 2008, 37, 912-920.

3. D. L. Jeanmaire and R. P. Vanduyne, J. Electroanal. Chem., 1977, 84, 120.

4. M. Moskovits, Rev. Mod. Phys., 1985, 57, 783-826.

5. A. G. Brolo, D. E. Irish and B. D. Smith, J. Mol. Struct., 1997, 405, 2944.

6. A. Campion and P. Kambhampati, Chem. Soc. Rev., 1998, 27, 241-250.

7. M. Moskovits, J. Raman Spectrosc., 2005, 36, 485-496.

8. T. Vo-Dinh, TrAC, Trends Anal. Chem., 1998, 17, 557-582.

9. C. Farcau and S. Astilean, Chem. Commun., 2011, 47, 3861-3863.

10. L. Guerrini and D. Graham, Chem. Soc. Rev., 2012, 41, 7085-7107.

11. M. Fan and A. G. Brolo, PCCP, 2009, 11, 7381-7389.

12. B. Sägmüller, B. Schwarze, G. Brehm and S. Schneider, Analyst, 2001, 126, 2066-2071.

13. S. P. Mulvaney, M. D. Musick, C. D. Keating and M. J. Natan, Langmuir, 2003, 19, 4784-4790.

14. A. C. De Luca, P. Reader-Harris, M. Mazilu, S. Mariggiò, D. Corda and A. Di Falco, ACS Nano, 2014, 8, 2575-2583.

15. S. Sriram, M. Bhaskaran, S. Chen, S. Jayawardhana, P. R. Stoddart, J. Z. Liu, N. V. Medhekar, K. Kalantar-Zadeh and A. Mitchell, J. Am. Chem. Soc., 2011, 134, 4646-4653.

16. S. Li, D. Wu, X. Xu and R. Gu, J. Raman Spectrosc., 2007, 38, 14361443.

17. S. Jayawardhana, G. Kostovski, A. P. Mazzolini and P. R. Stoddart, Appl. Opt., 2011, 50, 155-162.

18. K. T. Carron and L. G. Hurley, J. Phys. Chem., 1991, 95, 9979-9984.

19. A. A. Mani, Z. D. Schultz, Y. Caudano, B. Champagne, C. Humbert, L. Dreesen, A. A. Gewirth, J. O. White, P. A. Thiry and A. Peremans, $J$. Phys. Chem. B, 2004, 108, 16135-16138.

20. M. Moskovits and J. S. Suh, J. Phys. Chem., 1984, 88, 5526-5530.

21. R. Buividas, P. R. Stoddart and S. Juodkazis, Annalen der Physik, 2012, 524, L5-L10.

22. E. Sturrock, Q. Chen, P. Borchardt and N. Richardson, J. Electron. Spectrosc. Relat. Phenom., 2004, 135, 127-134.

23. J. Y. Gui, D. A. Stern, D. G. Frank, F. Lu, D. C. Zapien and A. T. Hubbard, Langmuir, 1991, 7, 955-963.

24. S. A. Claridge, J. J. Schwartz and P. S. Weiss, ACS Nano, 2011, 5, 693729.

25. B. M. Reinhard, S. Sheikholeslami, A. Mastroianni, A. P. Alivisatos and J. Liphardt, Proc. Natl. Acad. Sci. U. S. A., 2007, 104, 2667-2672. 